Patients avec un cancer de la prostate traités par hormonothérapie : Impact de l'Activité Physique Adaptée

Patients with prostate cancer treated by androgen deprivation therapy: Impact of adapted physical activity

\title{
LISTE DES AUTEURS
}

Veni Théoa ${ }^{a}$ Leroy Véronique ${ }^{b}$, Pradère Benjamin ${ }^{c d}$, Rébillard Amélie ${ }^{a}$, Mathieu Romain $^{\mathrm{b}}$

\author{
AFFILIATION \\ (a)Laboratoire « Mouvement, Sport, Santé » (EA 7470), Université Rennes, Campus \\ de Ker Lann, Bruz, France \\ (b)Service Urologie, Centre Hospitalier Universitaire De Rennes, France \\ (c)Service Urologie, CHRU Bretonneau, Tours, France \\ (d)Université François Rabelais de Tours, PRES Centre Val de Loir, Tours, France
}

\section{AUTEUR CORRESPONDANT}

Théo Veni

E-mail : theo.veni@aptitude-sante.fr

Phone: +33 (0) 625117476 


\section{Patients avec un cancer de la prostate traités par hormonothérapie : Impact de l'Activité Physique Adaptée}

\section{RÉSUMÉ}

Objectifs. Le but de cet article est de présenter l'Activité Physique Adaptée (APA) et son impact potentiel sur les effets secondaires de l'hormonothérapie (HT), les limites de sa mise en œuvre et les mesures pour favoriser sa diffusion auprès des patients avec un cancer de la prostate $(\mathrm{CaP})$ traité par HT.

Matériel et méthodes. Une revue non systématique de la littérature a été réalisée à partir des articles référencés dans Pubmed, en utilisant les mots clés " prostate cancer », « androgen deprivation » and "physical activity », et des principales publications et recommandations des agences de santé nationales et internationales, publiés entre Janvier 2010 et Juin 2019.

Résultats. L'APA représente un moyen d'action efficace pour diminuer les effets secondaires de l'HT. Son intégration dans le parcours de soins des patients avec un PCa traité par HT reste limitée.

Conclusion. Afin de promouvoir et garantir une place à l'APA dans cette population, une collaboration pluridisciplinaire entre les professionnels de santé et de l'APA est indispensable. Cette collaboration doit permettre la mise en place de programmes d'APA et d'outils d'éducation thérapeutique standards et innovants pour les patients ainsi que le développement d'information et de promotion auprès des professionnels de santé.

Mots clés : Cancer de la prostate, suppression androgénique, effets secondaires, activité physique adaptée, professionnels de santé 


\title{
Patients with prostate cancer treated by androgen deprivation therapy: Impact of adapted physical activity
}

\begin{abstract}
Objectives. The purpose of this article is to present Adapted Physical Activity (APA) and its potential impact on the androgen deprivation therapy (ADT) adverse effects, the limits and measures to promote its use among prostate cancer (PCa) patients treated with ADT.

Material and methods. A non-systematic review of the literature was performed with pubmed referenced articles, using the keywords "prostate cancer", "androgen deprivation" and "physical activity", and the main publications and recommendations of national and international health agencies, published between January 2010 and June 2019.
\end{abstract}

Results. APA represents an effective action to reduce adverse effects of ADT. Its integration into health care of PCa patients treated with ADT remains limited.

Conclusion. To promote a APA development in this population, a multidisciplinary collaboration between healthcare and APA professionals is essential. This collaboration should enable implementation of standard and innovative APA programs and therapeutic education tools for patients, as well as development of information and promotion for healthcare professionals.

\footnotetext{
Keywords. Prostate cancer, androgen deprivation, adverse events, physical activity, healthcare professional.
} 


\section{Patients avec un cancer de la prostate traités par hormonothérapie : Impact de l'Activité Physique Adaptée}

\section{INTRODUCTION}

Le traitement du cancer de la prostate $(\mathrm{CaP})$ peut nécessiter le recours à une hormonothérapie (HT). Selon les recommandations de l'Association Française d'Urologie, une HT peut être proposée en cas de CaP localisé de risque intermédiaire, de haut risque ou localement avancé, en association avec la radiothérapie, en cas de récidive après traitement d'un $\mathrm{CaP}$ localisé ou en cas de CaP métastatique(1). Ce traitement est à l'origine d'effets secondaires significatifs, tant physiques que psycho-sociaux. Ce traitement peut en effet être à l'origine de bouffées de chaleur, d'une gynécomastie, d'une prise de poids, d'une diminution de la force musculaire et d'une diminution de la densité minérale osseuse augmentant le risque de chutes et de fractures. L'augmentation de masse grasse et la diminution de masse musculaire, responsables de modifications métaboliques (résistance à l'insuline et altération du profil lipidique), conduisent à un syndrome « pseudométabolique ». Ce syndrome augmente le risque de pathologies cardiovasculaires, de troubles de l'humeur et diminue les capacités cognitives. Le patient peut également rapporter des troubles sexuels correspondant à une diminution du désir sexuel et/ou de l'excitation et une dysfonction érectile. Ces effets, associés au diagnostic récent du CaP et ses éventuels symptômes, diminuent considérablement la qualité de vie des patients. La prise en charge de ces effets secondaires potentiels fait donc l'objet de recommandations impliquant un bilan initial et un suivi régulier, l'éducation des patients et la mise en place de mesures(1). 
La promotion de l'APA pour les personnes atteintes d'un cancer quel que soit le traitement proposé est reconnue comme essentielle et inscrite dans plusieurs plans nationaux (e.g., Programme National Nutrition Santé 2017-2021, Plan cancer 2014-2019)(2,3). La mise en place et le maintien d'une activité physique dite adaptée (APA) est en effet une stratégie efficace pour limiter notamment les effets secondaires des traitements proposés. Dans le cadre du CaP, moins d'un patient sur deux atteint cependant le niveau d'activité physique recommandé(4). Dans ce contexte, la sensibilisation des patients ainsi que des professionnels de santé (PS) médicaux et paramédicaux à l'intérêt de l'APA dans l'offre de soins doit être encouragée.

Cet article présente l'APA et son impact potentiel sur les effets secondaires de l'HT, les limites de sa mise en œuvre et les mesures pour favoriser sa diffusion auprès des patients avec un CaP traité par HT.

\section{MATÉRIEL ET MÉTHODES}

Une revue non systématique de la littérature a été réalisée à partir des articles référencés dans Pubmed, en utilisant les mots clés " prostate cancer », " androgen deprivation » and "physical activity», et des principales publications et recommandations des agences de santé nationales et internationales, publiés entre Janvier 2010 et Juin 2019, afin de réaliser un article didactique sur les principes, les résultats et les limites de l'APA chez les patients avec un cancer de prostate traités par hormonothérapie. 


\section{RÉSULTATS ET DISCUSSION}

\section{Activité Physique Adaptée : Principes et bénéfices}

Des stratégies et recommandations ont été formulées pour lutter contre les effets secondaires de l'HT. En complément des bilans et suivis prescrits, la pratique d'une activité physique régulière est recommandée (1). L'activité physique se définit comme « tout mouvement corporel produit par contraction des muscles squelettiques entraînant une augmentation de la dépense énergétique par rapport à la dépense énergétique de repos »(5). D'après l'OMS, les activités physiques journalières peuvent être classées en quatre catégories (6) : les activités liées aux déplacements, les activités domestiques (e.g., bricolage, ménage), les activités professionnelles, et enfin, les activités de loisirs (e.g., sport). Lorsque ces activités sont «adaptées» aux capacités de la personne à des fins notamment de prévention, de rééducation, et de réadaptation, on parle d'activité physique adaptée (APA). L'APA peut alors être caractérisée par plusieurs facteurs : la Fréquence, l'Intensité, le Type d'activité et le Temps de maintien de l'activité (FITT)(7). L'intensité est souvent représentée par le MET (Metabolic Equivalent-task) qui est une quantité ou un débit d'énergie qui correspond à la dépense d'énergie en kilocalorie par kilogramme de poids de corps et par heure. Les activités peuvent ainsi être classées en activités à intensité faible, modérée, élevée ou très élevée(4).

De nombreuses études et méta-analyse récentes, ont démontré que la pratique d'une APA améliore la qualité de vie spécifique des patients traités par HT pour un CaP $(8,9)$. L'activité physique agit ainsi en contre-mesure précoce puis comme un processus de réadaptation à plus long terme. De manière globale, la pratique d'APA, par le biais d'exercices aérobie et/ou de renforcement musculaire, améliore la performance physique des patients. L'APA augmente ainsi les capacités 
fonctionnelles des patients (performances chronométriques à la marche, mesure de force), améliore leur composition corporelle (augmentation de la masse maigre, diminution du tour de taille et du poids) et diminue le risque de chute et de fracture. Un programme d'APA à une intensité adéquate, quelque soit le mode, contribue également à réduire la fatigue et le stress lié à la maladie. Elle améliore également les fonctions sexuelles et cognitives. Les études sur les troubles de l'humeur sont plus rares et ne permettent pas, par conséquent, de conclure sur le bénéfice de l'APA sur ces symptômes.

Les programmes d'APA décrits dans ces différentes études sont hétérogènes. II n'existe pas de programme type (Fréquence, Intensité, Type d'activité et le Temps de maintien de l'activité optimaux) afin d'obtenir ces bénéfices. Une méta-analyse récente a conclu à l'absence de preuves suffisantes pour produire des directives spécifiques d'exercice pour les patients avec un $\mathrm{CaP}(10)$. Ceci implique donc l'adoption des directives générales destinées aux patients atteints de cancer, qui sont les mêmes que celles correspondantes à la population générale : pratique d'activité physique modérée à intense de $30 \mathrm{~min} / \mathrm{jour}, 5$ jours/semaine, ce qui équivaut à une activité moyenne de 12 à 15 MET.h/semaine (11). L'OMS indique qu'il est nécessaire de pratiquer chaque semaine, au minimum, 150 minutes d'activité d'endurance à intensité modérée ou 75 minutes à intensité soutenue, ou une combinaison équivalente des deux (6). Des périodes minimales d'activité de 10 minutes sont nécessaires pour être comptabilisées. Afin de simplifier les notions de $\mathrm{MET}, \mathrm{km} / \mathrm{h}$ ou autre pourcentage, qui sont des notions peu évidentes, des repères simples tels que l'essoufflement, la pénibilité (e.g., échelle de Borg) peuvent guider patients et PS afin de prendre conscience de l'intensité des pratiques et évaluer leur volume d'activité (Tableau 1). Des exercices de renforcement musculaire faisant 
intervenir les principaux groupes musculaires doivent également être réalisés au moins deux jours par semaine, tout comme des exercices d'assouplissement et, si besoin, d'équilibre (Tableau 2). La pratique d'une APA comporte donc le recours éventuels à des activités très diverses mais doit permettre au patient de développer quatre qualités : la capacité cardiorespiratoire, les fonctions musculaires, l'assouplissement articulaire (i.e., gain d'amplitude) et le maintien de l'équilibre $(4,6)$.

Depuis la loi de modernisation de notre système de santé en date du 26 janvier 2016, il est désormais possible pour les médecins de prescrire de l'APA dans le cadre du parcours de soins des patients atteints d'une affection longue durée (ALD) (4). A cet effet, un guide national et un référentiel spécifique « cancer » sont proposés par la Haute Autorité de Santé pour faciliter les prescriptions (12).

L'orientation du patient vers l'offre d'APA varie cependant selon les territoires, les établissements et les réseaux de soins. Le patient peut être mis en relation directe avec des professionnels de l'APA, ou orienté vers des associations ou des structures labélisées. Actuellement, les programmes d'APA ne font pas l'objet d'un remboursement par l'Assurance Maladie. Cependant, certaines mutuelles proposent une offre de remboursement allant jusqu'à $500 €$.

\footnotetext{
Activité Physique Adaptée : Limites et Actions spécifiques à envisager

Malgré les nombreux bénéfices que peut engendrer l'APA dans le cadre du $\mathrm{CaP}$, la proportion de patients considérés comme actifs reste relativement faible. Ainsi, moins de la moitié des patients répondent aux recommandations actuelles concernant l'Activité Physique (4). Plusieurs raisons peuvent expliquer ces difficultés d'adhésion et il est urgent de mettre en place des actions spécifiques pour permettre le développement de l'APA chez les patients avec un CaP.
} 
Les recommandations actuelles ne prennent pas en compte les capacités fonctionnelles de chaque individu. En prenant en compte les co-morbidités de certains patients, l'ensemble des exercices recommandés, les rendez-vous médicaux, les traitements et le poids de la maladie, ces recommandations peuvent paraître irréalistes. Pour adapter les recommandations à chaque patient, il est donc essentiel que le concept de spécificité et de surcharge progressive soit appliqué à tous les exercices et que les programmes soient discutés et personnalisées avec l'aide d'un professionnel de l'APA $(4,11)$. La mise en place d'un réseau de soins intégrant un professionnel de l'APA est donc indispensable.

Si les PS sont conscients de leur rôle dans la promotion de l'activité physique pour les patients atteints de cancer, ils ne le font pas pour autant de façon systématique. Ainsi, Selon Keogh et al., seuls 40\% des PS assurent cette promotion accompagnée ou non de recommandations en matière d'AP(13). Pourtant, plusieurs études ont démontré que les patients avec un $\mathrm{CaP}$ ont une activité globale supérieure s'ils reçoivent des recommandations des cliniciens les prenant en charge. A l'inverse, le manque de directives et de conseils donnés au patient constitue un frein à la pratique. Les conclusions de nombreuses études indiquent que les PS devraient donc recevoir une formation appropriée sur les informations à fournir en matière d'APA aux hommes atteints de CaP (14). Différents PS ont été interrogés sur les stratégies permettant d'optimiser le développement de l'APA. Une meilleure information sur l'offre de soins concernant l'APA ainsi que des outils d'aide et d'information en ligne pour informer les patients étaient plébiscités (15). La promotion de l'APA ainsi que la mise à disposition d'outils facilitant sa mise œuvre auprès des PS est donc nécessaire. 
Le manque de pratique physique parmi les hommes suivis pour un $\mathrm{CaP}$ peut également s'expliquer par plusieurs particularités spécifiques à cette population. Ainsi, les hommes seraient moins enclins à adhérer à l'APA et à changer leur comportement que les femmes, expliquant en partie les différences d'adhésion observées par rapport aux patientes suivies pour un cancer du sein (4). Par ailleurs, l'âge médian de diagnostic du CaP est de 71 ans. L'âge fait partie, tout comme les co-morbidités pré-existantes, des facteurs prédictifs négatifs indépendants d'adhésion à l'APA (12). II est donc nécessaire de favoriser l'adhésion des hommes avec un $\mathrm{CaP}$ par des mesures spécifiques. Plusieurs études ont démontré que l'adhésion des hommes atteints de CaP pouvait être facilitée par la mise en place de techniques de changement de comportement (i.e., en anglais, " behaviour change techniques») (16). Bien que leur nombre soit conséquent, ce n'est pas la quantité de techniques utilisées mais la qualité qui est importante. D'après la revue d'Hallward et al., les études sur le CaP qui décrivent une meilleure adhésion des patients et une augmentation du niveau d'AP dans le temps comprennent un soutien social (en anglais, « social support »), la mise en œuvre d'informations sur l'activité physique et de rappels de pratique aux patients(17). Le soutien social est apporté à la fois par la présence d'un professionnel de l'APA mais également d'un groupe lors de séances d'exercice physique. La pratique structurée et encadrée fournit ainsi une motivation supplémentaire à l'exercice. La pratique libre "à domicile » trouve cependant de l'intérêt pour certains patients si un lien est gardé par mail ou suivi téléphonique. La souplesse de cette formule appuyée par des brochures ou des livrets d'exercices favorise sa pratique. Tous ces outils doivent cependant être maniés par des professionnels compétents (i.e., de l'APA ou de santé) pour être perçus comme des sources fiables par le patient. Toutefois, il est courant de s'apercevoir que les 
patients diminue leur quantité d'AP avec le temps. l'auto surveillance (en anglais, " self monitoring »), notamment par le développement d'outils connectés, etl'éducation thérapeutique du patient sont donc des notions très importantes pour permettre le maintien à long terme du niveau d'APA. Ces éléments imposent donc la mise en place de programmes d'APA intégrant les professionnels de santé et de l'APA, des outils d'information et de suivi des patients.

\section{CONCLUSION}

L'APA représente un moyen d'action efficace pour diminuer les effets secondaires de l'HT. Son intégration dans le parcours de soins des patients avec un PCa traité par HT reste limitée. Afin de promouvoir et garantir une place à l'APA dans cette population, une collaboration pluridisciplinaire entre les professionnels de santé et de l'APA est indispensable. Cette collaboration doit permettre la mise en place de programmes d'APA et d'outils d'éducation thérapeutique standards et innovants pour les patients ainsi que le développement d'information et de promotion auprès des PS.

\section{Conflits d'intérêt}

TV, VL et AR déclarent ne pas avoir de conflits d'intérêts.

RM : Consultant pour Bouchara-Recordati, Astra-Zeneca, Sanofi, Takeda, Janssen, Ipsen, Astellas, MSD et Investigateur pour APHP, Hôpitaux Civil de Lyon, Roche, Clinique Pasteur.

BP : Consultant pour Janssen, Ipsen. 
1. [French ccAFU guidelines - Update 2018-2020: Prostate cancer].

Rozet F, Hennequin C, Beauval JB, Beuzeboc P, Cormier L, Fromont-Hankard G, Mongiat-Artus P, Ploussard G, Mathieu R, Brureau L, Ouzzane A, Azria D, Brenot-Rossi I, Cancel-Tassin G, Cussenot O, Rebillard X, Lebret T, Soulié M, Penna RR, Méjean A. Prog Urol. 2018 Nov;28(12S):S79-S130.

2. Agence nationale de sécurité sanitaire de l'alimentation de l'environnement et du travail (ANSES). Actualisation des repères du PNNS - Révisions des repères relatifs à l'activité physique et à la sédentarité. 2016:420.

3. Institut National du Cancer. Plan cancer 2014 - 2019. 2015:1-8. papers://85259ad2-35a5-40eb-aed8-58d9d85dc63d/Paper/p76.

4. INCa. Bénéfices de l'activité Physique Pendant et Après Cancer: Des Connaissances Aux Repères Pratiques. Collection. (INCa, ed.). BoulogneBillancourt: INCa; 2017.

5. Caspersen CJ, Powell KE, Christenson GM. Physical activity, exercise, and physical fitness: definitions and distinctions for health-related research. Public Health Rep. 1985;100(2):126-131. doi:10.2307/20056429.

6. OMS. Recommandations Mondiales Sur L'Activité Physique Pour La Santé. Ed l'OMS. 2010:1-58. doi:10.1007/s13398-014-0173-7.2.

7. Barisic A, Leatherdale ST, Kreiger N. Importance of frequency, intensity, time and type (FITT) in physical activity assessment for epidemiological research. Can J Public Heal. 2011;102(3):174-175.

8. Gardner JR, Livingston PM, Fraser SF. Effects of exercise on treatmentrelated adverse effects for patients with prostate cancer receiving androgendeprivation therapy: A systematic review. J Clin Oncol. 2014;32(4):335-346. doi:10.1200/JCO.2013.49.5523. 
9. Yunfeng $G$, Weiyang $H$, Xueyang $H$, Yilong $H$, Xin G. Exercise overcome adverse effects among prostate cancer patients receiving androgen deprivation therapy: An update meta-analysis. Medicine (Baltimore). 2017;96(27):e7368. doi:10.1097/MD.0000000000007368.

10. Owen PJ, Daly RM, Livingston PM, Fraser SF. Lifestyle guidelines for managing adverse effects on bone health and body composition in men treated with androgen deprivation therapy for prostate cancer: An update.

Prostate Cancer Prostatic Dis. 2017;20(2):137-145. doi:10.1038/pcan.2016.69.

11. Agence nationale de sécurité sanitaire de l'alimentation de l'environnement et du travail (ANSES). Actualisation des repères du PNNS - Révisions des repères relatifs à l'activité physique et à la sédentarité. 2016:420.

12. Haute Autorité de Santé. Guide de promotion, consultation et prescription médicale d'activité physique et sportive pour la santé chez les adultes, juillet 2019.

13. Keogh JWL, Olsen A, Climstein M, Sargeant S, Jones L. Benefits and Barriers of Cancer Practitioners Discussing Physical Activity with their Cancer Patients. J Cancer Educ. 2017;32(1):11-15. doi:10.1007/s13187-015-0893-1.

14. Sutton E, Hackshaw-McGeagh LE, Aning J, et al. The provision of dietary and physical activity advice for men diagnosed with prostate cancer: a qualitative study of the experiences and views of health care professionals, patients and partners. Cancer Causes Control. 2017;28(4):319-329. doi:10.1007/s10552017-0861-7.

15. Cantwell M, Walsh D, Furlong B, et al. Healthcare professionals' knowledge and practice of physical activity promotion in cancer care: Challenges and 
solutions. Eur J Cancer Care (Engl). 2017;(October):e12795. doi:10.1111/ecc.12795

16. Michie S, Richardson M, Johnston M, et al. The behavior change technique taxonomy (v1) of 93 hierarchically clustered techniques: Building an international consensus for the reporting of behavior change interventions. Ann Behav Med. 2013;46(1):81-95. doi:10.1007/s12160-013-9486-6.

17. Hallward L, Patel N, Duncan LR. Behaviour change techniques in physical activity interventions for men with prostate cancer: A systematic review. $J$ Health Psychol. 2018. doi:10.1177/1359105318756501. 
Tableau 1 Repères de pratique de l'activité physique pour les patients atteints de cancer de la prostate (selon l'INCa, 2017)

\begin{tabular}{|c|c|c|c|}
\hline Intensité & Mesures & Repères d'intensité & Exemples \\
\hline & & - Pas d'essoufflement & - Marche $<5 \mathrm{~km} / \mathrm{h}$ \\
\hline Faible & 1,6 à 2,9 MET & $\begin{array}{l}\text { - Pas de transpiration } \\
\text { - Pénibilité 2-4 }\end{array}$ & $\begin{array}{l}\text { - Promener son chien } \\
\text { - Conduite automobile }\end{array}$ \\
\hline & & $\begin{array}{l}\text { - Essoufflement modéré } \\
\text { - Conversation possible }\end{array}$ & $\begin{array}{l}\text { - Marche de } 5 \text { à } 6,5 \text { km/h } \\
\text { - Montée d'escaliers à }\end{array}$ \\
\hline Modérée & 3 à 5,9 MET & $\begin{array}{l}\text { - Pénibilité 5-6 } \\
\text { - Peut être maintenue } 30 \text { à } \\
60 \text { minutes }\end{array}$ & $\begin{array}{l}\text { vitesse lente } \\
\text { - Vélo à } 15 \mathrm{~km} / \mathrm{h}\end{array}$ \\
\hline Élevée & 6 à 8,9 MET & $\begin{array}{l}\text { - Essoufflement } \\
\text { - Conversation difficile } \\
\text { - Transpiration abondante } \\
\text { - Pénibilité 7-8 } \\
\text { - Ne peut être maintenue } \\
\text { plus de } 30 \text { minutes }\end{array}$ & $\begin{array}{l}\text { - Marche à } 5 \mathrm{~km} / \mathrm{h} \text { sur } \\
\text { une pente à } 12 \% \\
\text { - Montée rapide } \\
\text { d'escaliers } \\
\text { - Vélo à } 20 \mathrm{~km} / \mathrm{h} \\
\text { - Course de } 8 \text { à } 9 \mathrm{~km} / \mathrm{h}\end{array}$ \\
\hline
\end{tabular}

MET : metabolic equivalent task, équivalent métabolique 
Tableau 2 Recommandations de pratique de l'activité physique pour les patients atteints de cancer de la prostate(selon l'INCa, 2017 et la revue d'Owen, 2017)

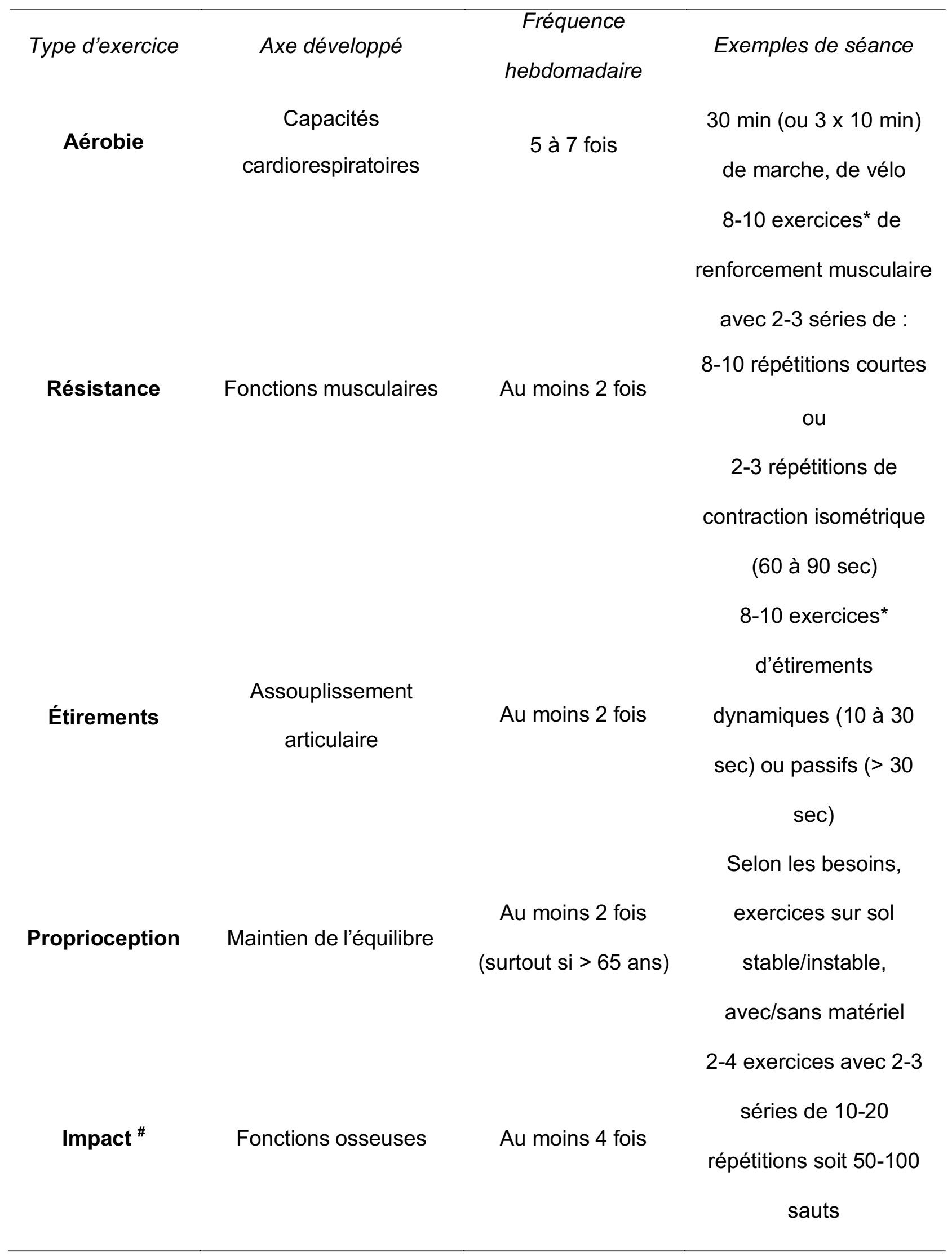


*ciblant les principaux groupes musculaires, en particulier les muscles des membres

inférieurs et de la région abdomino-dorsale

\# Il est recommandé de réaliser des exercices de résistance préalables pour ceux qui ontune

faible force musculaire et/ou une fonction musculaire faible avant de commencer certaines activités d'impact. 